
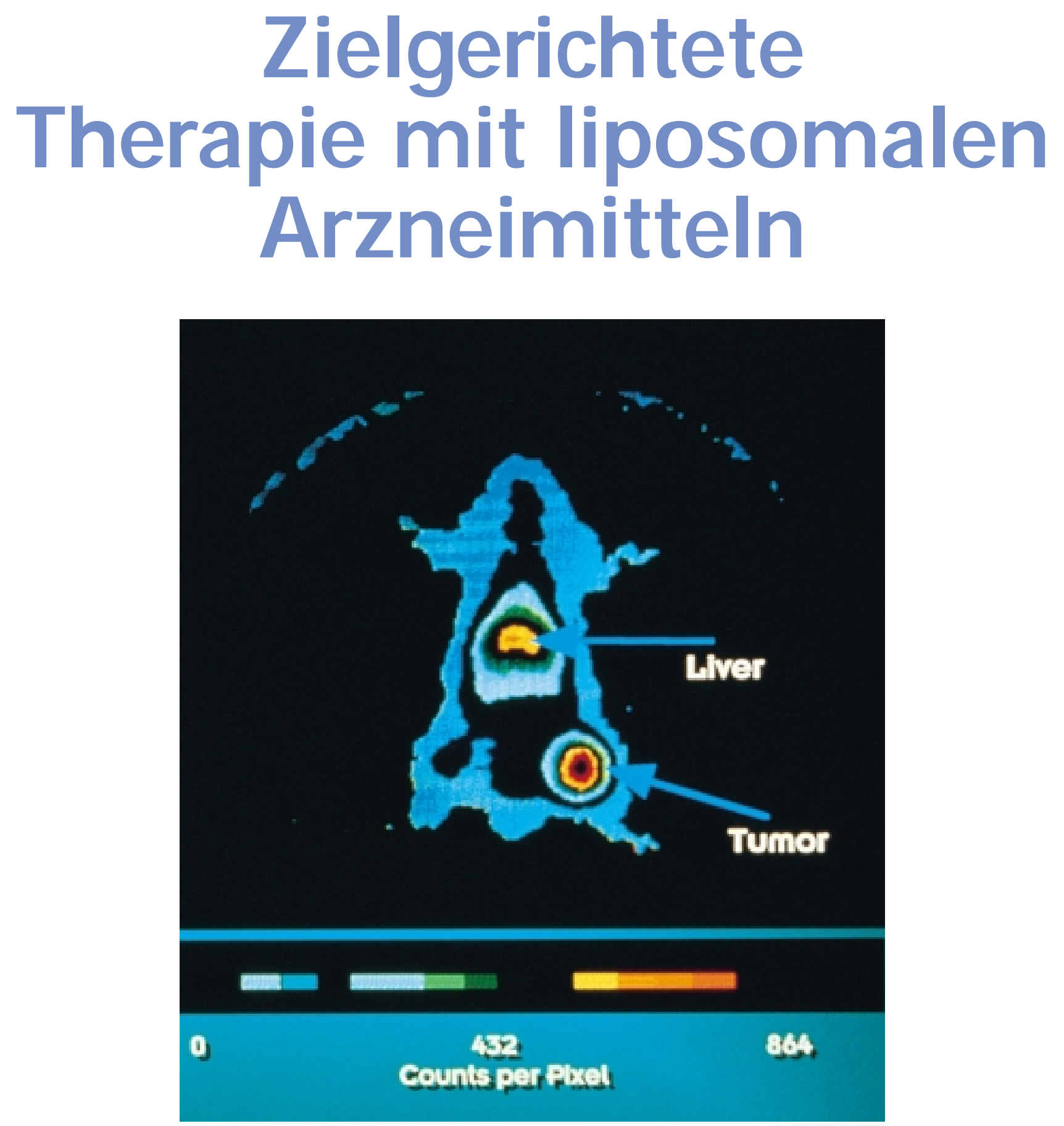

Gamma camera image of tumor-bearing mouse injected with liposome-entrapped Indium-111

$M$ it freundlicher U nterstützung durch

GILEA D Sciences, M ünchen

S. Karger

M edical and Scientific

Publishers

B asel · Freiburg

Paris . London

New York - New D elhi

B angkok · Singapore

Tokyo $\cdot$ Sydney
KARGER

Beilage zu ONKOLOGIE Band 24, Heft 1, Februar 2001

International Journal for
Cancer Research and Treatment

(c) Copyright 2001 by S. Karger G mbH, D-79095 Freiburg 


\section{Zielgerichtete Therapie mit liposomalen Arzneimitteln}

\section{Die Vorteile: selektiv - gut verträglich - wirksam}

Wie erfolgreich eine Therapie ist, hängt von ihrem therapeutischen Index ab. D abei werden Wirksamkeit und Verträglichkeit zueinander in Beziehung gesetzt. $E$ in hoher therapeutischer Index steht für gute Wirksamkeit und geringe Nebenwirkungen. E ine vielversprechende Möglichkeit, den therapeutischen Index zu erhöhen, ist die liposomale Verkapselung des Wirkstoffes. E rst am W irkort wird dieW irksubstanz freigesetzt, um dort - ganz gezielt - die therapeutische Wirkung zu entfalten. Dieser sehr spezifische Therapieansatz reduziert die Nebenwirkungen und scheint zu höheren E rfolgsraten zu führen.

E ine der ersten Substanzen, die liposomal appliziert wurde, ist das $B$ reitband-A ntimykotikum $A$ mphotericin $B$, das als $A \mathrm{mB}$ isome ${ }^{\circledR}$ zur Behandlung schwerer systemischer Pilzerkrankungen zugelassen ist. A mphotericin $B$ ist für diese Indikation Therapie der Wahl.

Problematisch sind allerdings die zum Teil schweren $\mathrm{N}$ ebenwirkungen von konventionell appliziertem A mphotericin B, die den Therapieerfolg limitieren. Im Vordergrund steht die Nephrotoxizität. A mBisome hat aufgrund der liposomalen Verkapselung des Wirkstoffes viele der A mphotericin-Btypischen Nebenwirkungen nicht oder signifikant seltener. $\mathrm{K}$ linische Studien bestätigen, dass $\mathrm{A} \mathrm{mB}$ isome hohe $\mathrm{D}$ osen des Wirkstoffes A mphotericin B direkt (selektiv) an den zu bekämpfenden Pilz abgibt.

\section{Liposomale Verkapselung von Daunorubicin}

Mittlerweile wurden auch für die A nthrazykline liposomale Transport- und A bgabesysteme entwickelt. A nthrazykline gehören zu den wirksamsten Zytostatika bei zahlreichen Tumorerkrankungen, z. B. bei der akuten myeloischen Leukämie (AML) oder bei Non-Hodgkin-Lymphomen (NHL). Die A nthrazykline Daunorubicin und D oxorubicin sind hier die E ckpfeiler der Therapie.

Auch der Therapieerfolg der A nthrazykline wird durch zum Teil sehr ausgeprägte akute Toxizitäten limitiert. Speziell die kumulative kardiotoxische Wirkung kann zu schweren, teilweise lebensbedrohlichen kardialen Komplikationen führen. Weitere klinisch relevante Nebenwirkungen der Anthrazykline sind die Knochenmarksuppression, gastrointestinale Störungen und lokale G ewebsnekrosen. Subjektiv stark belastend - speziell für viele Frauen - ist die A lopezie.

$M$ it der liposomalen Verabreichung von $D$ aunorubicin (D aunoX ome ${ }^{\circledR}$ ) ist es jedoch gelungen, die hohe antineoplastische Potenz der A nthrazykline therapeutisch zu nutzen und die Anthrazyklin-typischen Nebenwirkungen deutlich zu reduzieren.

Klinisch wurde DaunoXome zunächst bei Patienten mit A ID S-bedingtem $K$ arposisarkom eingesetzt und ist für diese Indikation auch zugelassen. $\mathrm{H}$ ier erreicht die Substanz selbst bei Hochrisikopatienten gute A nsprechraten. Die körperliche Leistungsfähigkeit der Patienten bleibt während der Therapiedauer erhalten. $\mathrm{K}$ linisch relevante $\mathrm{K}$ ardiotoxizitäten treten nicht auf, die linksventrikuläre A uswurfsfraktion (LVEF) bleibt unverändert. Nichthämatologische Neben-

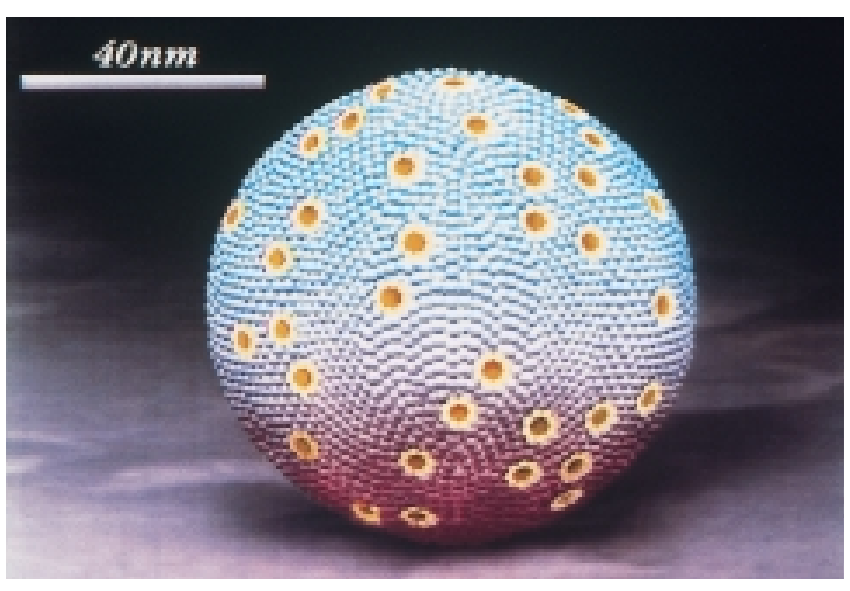

Abb. 1 D as A mB isome-L iposom - A ußenansicht (Schema).

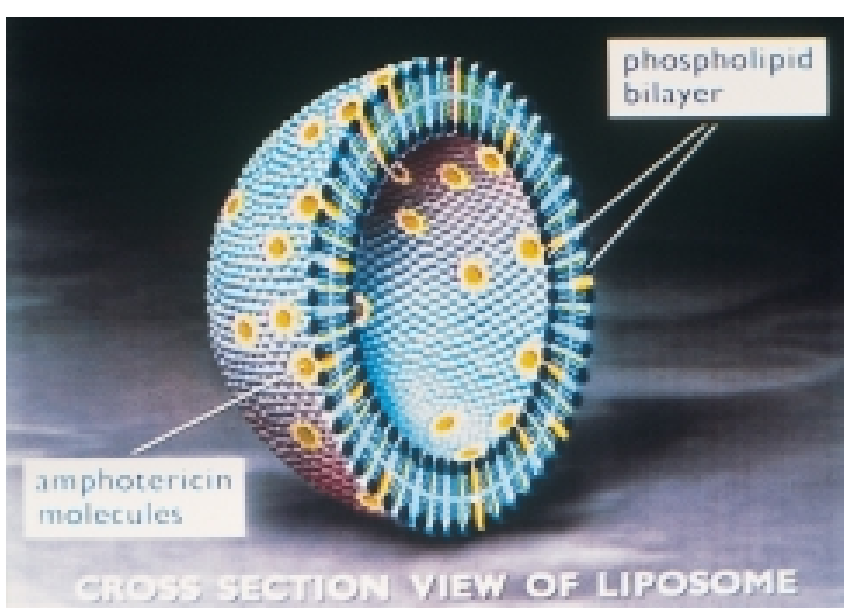

Abb. 2. Schnitt durch das A mB isome-Liposom (Schema).

wirkungen sind selten und nur gering ausgeprägt. E rste vielversprechende klinische $\mathrm{E}$ rgebnisse mit $\mathrm{D}$ aunoX ome liegen mittlerweile auch bei Patienten mit A ML und NHL vor.

\section{Ein ausgeklügeltes Transport- und A bgabesystem}

U $\mathrm{m}$ im menschlichen $\mathrm{K}$ örper als gezieltes Transportvehikel wirksam zu sein, müssen Liposome einen Balanceakt vollbringen: Sie müssen intakt bleiben, während sie zirkulieren, und sich auflösen, um den Wirkstoff freizusetzen, sobald sie den Wirkort erreicht haben. Bei der Entwicklung von $A \mathrm{mB}$ isome und $\mathrm{D}$ aunoX ome wurde dies durch ein ausgeklügeltes Transportsystem erreicht.

Die unilamellaren L iposomen sind relativ klein (D urchmesser 35- $65 \mathrm{~nm}$ ). D amit haben sie gegenüber größeren Liposomen eine deutlich verlängerte $Z$ irkulationszeit. G leichzeitig sind sie zu groß, um normal endothelialisierte $G$ efäßwände zu verlassen und so in den systemischen $\mathrm{K}$ reislauf und damit in gesunde $O$ rgansysteme zu gelangen (A bb. 1).

Die Lipidmembran der Liposome besteht im Wesentlichen aus Phospholipiden und eingelagertem Cholesterin. D as 


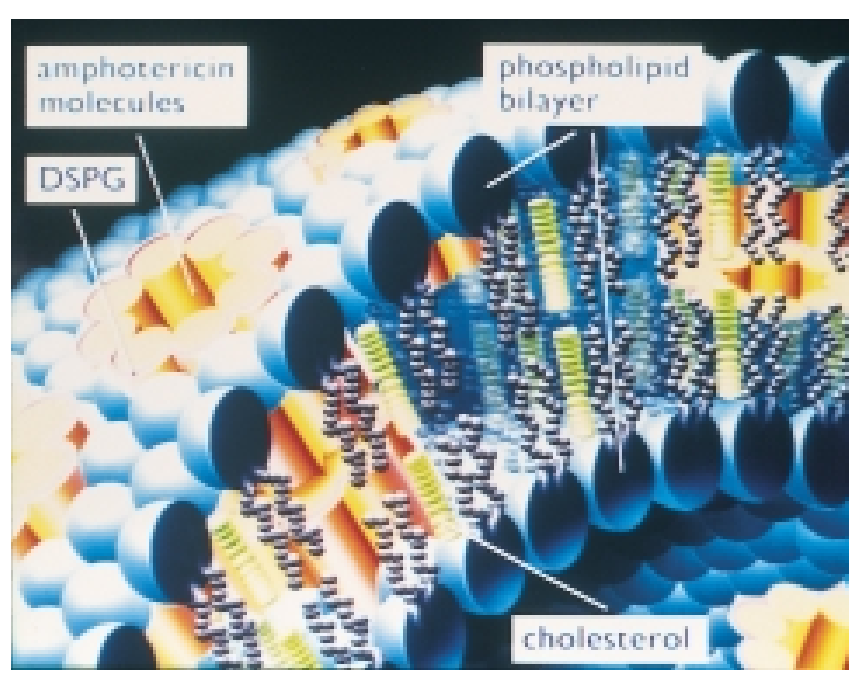

Abb. 3. A ufbau der L iposomenwand von A mB isome (Schema).

Cholesterin verstärkt die strukturelle Festigkeit des L iposoms und vermindert zusätzlich den Substanzverlust. D iese Z usammensetzung garantiert al so eine hohe Stabilität im B lut und ist damit ebenfalls ein wesentlicher Faktor für die geringe systemische Toxizität (A bb. 2).

A mB isome ist das erste intravenös verabreichte liposomale M edikament auf dem M arkt. Der Wirkstoff, das A mphotericin B, ist in die Doppelschichtmembran des unilamellären Liposoms integriert (A bb. 3). A mB isome lagert sich an den pilzinfizierten Stellen an. Durch die direkte Interaktion mit der Pilzzelle kommt es zum Zerfall des Liposoms und zur Freisetzung von A mphotericin $B$.

B eim DaunoX ome ist der Wirkstoff, das Daunorubicin, im wässerigen Innenraum des unilamellaren Liposoms eingeschlossen. In Vergleichsuntersuchungen zeigte sich, dass die A bgabe desW irkstoffes höher und die Wirksamkeit besser ist, wenn Daunorubicin im wässerigen Innenraum statt in der M embrandoppelschicht eingeschlossen ist. Der Grund dafür ist die erhöhte Stabilität im Plasma und damit ein hoher Schutz vor Substanzverlust auf dem Transportweg zum Tumor.

$D$ ie DaunoX ome-Liposome sind klein genug, um durch das poröse Endothel im Tumor zu diffundieren. E s ist bekannt, dass die K apillaren im Tumor eine andere Z usammensetzung haben und für kleine Partikel durchlässiger sind. Die Lipidmembran des Liposoms besteht aus Distearoylphosphatidylcholin und Cholesterol im molaren Verhältnis von 2:1. $D$ iese Kombination zeigte in vivo eine besonders hohe A ffinität zu soliden Tumoren. Klinisch führt dies zu einer verstärkten A kkumulation des Zytostatikums im Tumorgewebe und zu einer reduzierten Toxizität.

\section{Veränderte Pharmakokinetik unterstützt Wirksamkeit derTherapie}

D urch die liposomale Formulierung wird die Pharmakokinetik von A mphotericin B und D aunorubicin verändert. I $m$ Vordergrund stehen höhere Serum-Spitzenkonzentrationen und eine längere Eliminations-H albwertszeit. Letztere beträgt unter einer D osierung von 3 und $5 \mathrm{mg} / \mathrm{kg} \mathrm{A} \mathrm{mB}$ isome $26-38 \mathrm{~h}$. D ie einmal tägliche Injektion von A mB isome ist damit in der L age, konstante therapeutische Konzentrationen aufrecht zu erhalten.

A uch D aunoX ome erreicht in der Tumorzelle lang anhaltende Plasmaspiegel von Daunorubicin. Die humane Pharmakokinetik zeigt höhere Plasmaspiegel und eine verminderte Clearance- $R$ ate von liposomalem $D$ aunorubicins im Vergleich zur konventionell gegebenen Substanz (Tab. 1). In Laboruntersuchungen erreicht die Fluoreszenz des freien Daunorubicin nach 28-32 h das $M$ aximum, normales $G$ ewebe wird nur minimal beeinträchtigt. Konventionell verabreichtes D aunorubicin erreicht dagegen im Tumor und im umgebenden gesunden Gewebe nach wenigen Stunden mittlere Gewebespiegel, die rasch wieder abfallen. D ies deutet darauf hin, dass das liposomale Daunorubicin gegenüber konventionell verabreichtem Daunorubicin eine verstärkte zytotoxische A ktivität entfaltet, die über einen längeren Z eitraum anhält.

\section{DaunoXome in Phase-I-Studien zur Behandlung von Patienten mit rezidivierter bzw. refraktärer akuter AML}

Der Chemotherapie kommt bei der Behandlung der A M L zentrale B edeutung zu. D aunorubicin ist hier eines der wirksamsten Zytostatika. E tablierte Induktionstherapien sind das «7+3»-R egime (A raC/D aunorubicin) und das TA D -Protokoll (6-Thioguanin, A raC, D aunorubicin). N eu ist die Ü berlegung, durch eine Intensivierung der Chemotherapie den Therapieerfolg zu verbessern. Z iel ist es, die R ate der Langzeitremissionen zu erhöhen. Intensivere Chemotherapien, speziell höhere A nthrazyklin-D osen, steigern jedoch das R isiko der kumulativen $\mathrm{K}$ ardiotoxizität. $\mathrm{D}$ ies hat $\mathrm{A}$ uswirkungen auf die primäre Therapie und die Rezidivbehandlung. Besondere Bedeutung kommt der Behandlung älterer A M L -Patienten ( $\geq 60$ Jahre) zu. Sie weisen gegenüber jüngeren Patienten nicht nur verstärkt Komorbiditäten - z. B. kardiale Vorerkrankungen - auf, sondern haben auch eine prognostisch ungünstigere $\mathrm{K}$ rankheitsbiologie. Typisch sind beispielsweise krankheitsspezifische, für die Prognose ungünstige Chromosomenaberrationen, wie z.B. K aryotypanomalien sowie eine vermehrte Expression des MDR-1-G ens, das für die Chemotherapieresistenz verantwortlich gemacht wird. D iese und andere Veränderungen treten bei älteren Patienten häufiger auf als bei jüngeren. $G$ ünstige $A$ berrationen, die bei jüngeren Patienten mit einer hohen Heilungsrate einhergehen, kommen bei älteren Patienten fast nicht vor.

Vom liposomalen D aunorubicin ( $D$ aunoX ome) verspricht man sich eine bessere Verträglichkeit der B ehandlung, ohne dass die Wirksamkeit abnimmt. Präklinische U ntersuchungen lassen auch eine höhere $A$ ktivität vermuten, denn D aunoX ome scheint PGP(P-Glykoprotein)-vermittelte R esistenzmechanismen zu überwinden. Prof. D r. med. Thomas B üchner, U niversität $M$ ünster, stellte die wichtigsten präklinischen und erste klinische Daten von D aunoX ome vor, unter anderem die Ergebnisse zweier Phase-I-Studien bei Patienten mit refraktärer bzw. rezidivierter A M L. In beiden Studien waren die Patienten älter als 60 Jahre. Im M ittelpunkt stand jeweils die Verträglichkeit. 
Tab. 1 Pharmakokinetische Veriablen von freiem Daunorubicin im Vergleich

\begin{tabular}{lcccc}
\hline & $\mathrm{T}_{1 / 2}, \mathrm{~h}$ & $\mathrm{~A} \cup \mathrm{C},(\mathrm{ng} \cdot \mathrm{h}) / \mathrm{ml}$ & $\mathrm{Cl}, \mathrm{ml} / \mathrm{min}$ & $\mathrm{Vd}, \mathrm{I}$ \\
\hline Freies & & & & \\
D aunorubicin & 4,75 & 6253 & 247 & 89,7 \\
DaunoX ome & 5,08 & 61756 & 21,1 & 9,34 \\
\hline $\mathrm{Cl}=$ Clearance; $\mathrm{Vd}=$ Verteilvolumen. & & \\
\hline
\end{tabular}

\section{Phase-I-Studie an der Universitätsklinik Münster: Liposomales Daunorubcin reduziert die Nebenwirkungen deutlich}

In den eigenen Phase-I-Studien waren 14 Patienten mit einem medianen A Iter von 69 Jahren und einem Karnofsky-Index von (M edianwert) 70 (50-90) aufgenommen worden. Es handelte sich um Hochrisikopatienten, für die nur noch eine palliative Behandlung in Frage kam. Elf Patienten hatten einen Relapse, 3 Patienten waren refraktär, 5 Patienten zeigten komplexe K aryotypanomalien.

Die Patienten waren intensiv vorbehandelt. Die kumulative D aunorubicin-D osis aus der vorangegangenen B ehandlung betrug im M edian $670 \mathrm{mg} / \mathrm{m}^{2}$, lag also sehr hoch, und ging in E inzelfällen bis $880 \mathrm{mg} / \mathrm{m}^{2} \mathrm{D}$ aunorubicin. D ies bedeutete ein deutlich erhöhtes kardiales R isiko.

D aunoX ome wurde in vier D osisstufen appliziert, beginnend mit $40 \mathrm{mg} / \mathrm{m}^{2}$ alle 14 Tage. Traten keine dosislimitierenden $\mathrm{N}$ ebenwirkungen auf, wurde die D osis auf 60,75 und schließlich auf $90 \mathrm{mg} / \mathrm{m}^{2}$ erhöht. Im Mittel hatten die Patienten nach Studienende eine kumulative Dosis von $340 \mathrm{mg} / \mathrm{m}^{2}$ Dauno$X$ ome erhalten.

Die nichthämatologischen N ebenwirkungen waren nur leicht bis moderat ausgeprägt und damit gut tolerabel. Im Vordergrund stand die Fatigue (6/14 Patienten), gefolgt von Ü belkeit/E rbrechen. K ein Patient litt an M ukositis oder A lopezie. Nur 1 Patient hatte eine leichte D iarrhöe (Tab. 2).

$D$ as wichtigste E rgebnis der Studie ist laut Büchner, dass bei keinem Patienten eine klinisch relevante K ardiotoxizität auftrat. K ein Patient zeigte eine Veränderung der LVEF. Dies ist umso bemerkenswerter, als viele Patienten intensiv mit Daunorubicin vorbehandelt waren und auch während der Studie zum Teil hohe DaunoX ome-Dosen erhalten hatten. Tabelle 3 zeigt die Ergebnisse im Detail bei 6 kardiologisch vollständig ausgewerteten Patienten.

Drei der 14 Patienten erreichten eine partielle Remission, definiert als $\mathrm{A}$ bnahme der leukämischen Blasten im $\mathrm{K}$ nochenmark um mehr als $25 \%$. B ei einem weiteren Patienten stabilisierte sich die E rkrankung. 3 Patienten waren progredient. 7 Patienten starben innerhalb von 6 Wochen, meist an Infektionen (Tab. 4).

\section{Phase-I-Studie vom M.D. Anderson Cancer Center, Houston: Gute Verträglichkeit und antileukämische Aktivität}

D ie zweite Phase-I-Studie wurde am M . D. A nderson Cancer Center in H ouston/U SA durchgeführt (Cortes et al., 1999). A ufgenommen wurden 23 Patienten, ebenfalls mit rezidivier-
Tab. 2. Nichthämatologische Nebenwirkungen unter DaunoX omeMonotherapie bei stark vorbehandelten AML-Patienten $(n=14)$, Phase-I-E rgebnisse (nach Th. Büchner, M ünster)

\begin{tabular}{lll}
\hline \multirow{2}{*}{ N ebenwirkungen (G rad 1-2) } & \multicolumn{2}{l}{ Patienten } \\
\cline { 2 - 3 } & $\mathrm{n}$ & $\%$ \\
\hline Fatigue & $6 / 14$ & 50 \\
N ausea & $5 / 14$ & 43 \\
G ewichtsverlust & $4 / 14$ & 36 \\
Fieber & $4 / 14$ & 36 \\
H ospitalisierung & $4 / 14$ & 36 \\
i. v. A ntibiotikatherapie & $3 / 14$ & 31 \\
A nstieg der Leberenzyme & $2 / 14$ & 21 \\
N europathie & $2 / 14$ & 15 \\
D iarrhöe & $1 / 14$ & 7 \\
A lopezie & $0 / 14$ & 0 \\
M ukositis & $0 / 14$ & 0 \\
\hline
\end{tabular}

ter bzw. refraktärer A M L. Im U nterschied zur M ünsteraner Studie wurden deutlich höhere $D$ aunoX ome-D osen gegeben. Initial erhielten die Patienten $75 \mathrm{mg} / \mathrm{m}^{2}$ D aunoX ome an 3 aufeinander folgenden Tagen. Traten keine dosislimitierenden Nebenwirkungen auf, so stieg die D osis von 100 auf 150, dann auf $200 \mathrm{mg} / \mathrm{m}^{2}$ D aunoX ome.

17 der 23 Patienten waren mit A nthrazyklinen vorbehandelt. 15 Patienten erhielten einen Therapiekurs mit $D$ aunoX ome, 7 Patienten zwei und 1 Patient drei Therapiekurse.

Die Ergebnisse zeigt Tabelle 5. Dosislimitierend war die M ukositis ( $\mathrm{Grad}$ 3-4) bei 3 von 5 Patienten auf der höchsten D osisstufe ( $200 \mathrm{mg} / \mathrm{m}^{2} \mathrm{D}$ aunoX ome, Tage 1-3). A uch in dieser Studie kam es trotz der deutlich höheren Dosierung auf keiner D osisstufe zu klinisch relevanter $\mathrm{K}$ ardiotoxizität oder zu einer Veränderung der LVE F.

$Z$ wei der 23 Patienten erreichten eine komplette R emission: Im ersten Fall handelte es sich um eine CM L-B lastenkrise, die in eine chronische Phase überging. Beim zweiten Patienten handelte es sich um ein Mehrfachrezidiv einer Promyelozytenleukämie, die unter der DaunoX ome-M onotherapie in eine komplette R emission zurückgeführt wurde.

Als maximal tolerable Dosis definierten die Autoren $150 \mathrm{mg} / \mathrm{m}^{2}$ DaunoX ome an 3 konsekutiven Tagen. Diese Dosierung wurde gut vertragen und zeigte eine gute antileukämische Wirksamkeit.

Fazit: D ie M onotherapie mit D aunoX ome bei älteren Patienten mit refraktärer bzw. rezidivierter $A M L$ ist trotz zum Teil äußerst ungünstiger $R$ andbedingungen gut verträglich. Weitere Studien sind notwendig, um die optimale Dosis zu definieren und in diesem Zusammenhang auch die zytotoxische A ktivität zu evaluieren. A ufgrund der guten Verträglichkeit bietet sich $\mathrm{D}$ aunoX ome für die Kombinationsbehandlung in den früheren E rkrankungsstadien an.

\section{Mit DaunoXome Chemotherapieresistenz überwinden}

In-vitro-D aten deuten darauf hin, dass DaunoX ome neben der guten Verträglichkeit auch in der Lage ist, eine Chemotherapieresistenz zu überwinden. Büchner verwies auf eine Untersuchung von M ichieli et al., U niversität U dine [1999]. 
Tab. 3. K ardiale Toxizität unter D aunoX ome-M onotherapie bei stark vorbehandelten A M L -Patienten; Phase-I-E rgebnisse (nach Th. Büchner, M ünster)

\begin{tabular}{llllll}
\hline $\begin{array}{l}\text { Patient } \\
\text { Nr. }\end{array}$ & LVEF, & $\begin{array}{l}\text { A nzahl der } \\
\text { Therapiekurse }\end{array}$ & $\begin{array}{l}\text { DaunoX ome- } \\
\text { Dosierung } \\
\mathrm{mg} / \mathrm{m}^{2}\end{array}$ & $\begin{array}{l}\text { Vorbehandlung } \\
\text { mit Daunorubicin } \\
\mathrm{mg} / \mathrm{m}^{2}\end{array}$ \\
\hline $1^{\text {a }}$ & 67 & 52 & 3 & 40 & 740 \\
2 & 58 & 60 & 8 & 40 & 740 \\
3 & 51 & 60 & 11 & 60 & 300 \\
$4^{\text {b }}$ & 58 & 46 & 4 & 60 & 0 \\
5 & 67 & 65 & 7 & 75 & 675 \\
6 & 50 & 53 & 3 & 75 & 675 \\
\hline
\end{tabular}

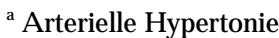

${ }^{\mathrm{b}}$ Vorbestehende K HK plus M yokardinfarkte.
Tab. 4. A ktivität der $D$ aunoX ome-M onotherapie bei stark vorbehandelten A M L -Patienten; Phase-I -E rgebnisse (nach Th. B üchner, M ünster)

\begin{tabular}{llr}
\hline & \multicolumn{2}{l}{ Patienten } \\
\cline { 2 - 3 } & $\mathrm{n}$ & $\%$ \\
\hline Tumoransprechen $^{\mathrm{a}}$ & 3 & 21 \\
Tumorstabilisierung $^{\mathrm{b}}$ & 1 & 7 \\
Progress $_{\text {Tod }^{c}}$ & 3 & 21 \\
\hline
\end{tabular}

${ }^{a} A$ bnahme leukämischer Blasten im K nochenmark um $>25 \%$.

${ }^{\mathrm{b}} \mathrm{K}$ eine Veränderung der A nzahl leukämischer B lasten im

K nochenmark.

cInnerhalb von 6 Wochen.

D ie Studiengruppe applizierte DaunoX ome bzw. StandardD aunorubicin in A M L-Zell-Linien, darunter M ultidrug-resistente (M D R-1-Träger) und nicht resistente Zell-Linien.

$B$ ei den nicht resistenten Z ellen ergaben sich keine zytotoxischen Unterschiede zwischen DaunoX ome und freiem Daunorubicin. DaunoX ome zeigte jedoch eine deutlich höhere zytotoxische Aktivität bei den resistenten Zellen (M D R-1-Träger) als das freie D aunorubicin. Wahrscheinlich ist die verstärkte $A$ kkumulation von DaunoX ome in diesen Zellen dafür verantwortlich.

B eide Darreichungsformen wurden zusätzlich in humanen leukämischen Blasten untersucht. $\mathrm{H}$ ier zeigte sich eine Korrelation zwischen PG P-Expression und zytotoxischer Wirksamkeit. Bei niedriger PGP-Expression akkumulierte liposomal appliziertes Daunorubicin intrazellulär schneller und höher als konventionell appliziertes Daunorubicin. B ei hoher PG PE xpression war die $A \cup C$ beider $D$ arreichungsformen sehr ähnlich; die intrazelluläre A kkumulation von normalem Daunorubicin erreichte jedoch früher ein niedriges Plateau, während es unter der liposomalen $\mathrm{G}$ abe zu einem langsamen, aber stetigen A nstieg der D aunorubicin-Konzentration kam.

Michieli et al. kommen zu dem Schluss, dass liposomal verkapseltes $D$ aunorubicin vor PG P-Proteinen geschützt ist. A ußerdem akkumulieren die Liposome verstärkt in PGPüberexprimierenden leukämischen Blasten. Liposomales $D$ aunorubicin könnte damit eine interessante Therapiealternative speziell für Patienten mit PGP-positiver akuter L eukämie sein.
Tab. 5. E rgebnisse nach D anoX ome-M onotherapie, $100-200 \mathrm{mg} / \mathrm{m}^{2}$, an 3 konsekutiven Tagen. U S-amerikanische Phase-I-Studie (nach Th. B üchner, M ünster)

\section{A nzahl}

Patienten

$\begin{array}{ll}\text { Toxizität } & \\ \text { M ukositis G rad 3-4 = dosislimitierend } & \\ \text { D osis, } \mathrm{mg} / \mathrm{m}^{2} & 3 / 5 \\ 200 & 2 / 9 \\ 150 & 1 / 6 \\ 100 & \\ \text { K eine K ardiotoxizität } & 0 / 9 \\ \text { LV E F-V eränderungen } & 0 / 23 \\ \text { Signifikante kardiale E reignisse } & \end{array}$

A nsprechen

Komplette R emission

Schlußfolgerung der A utoren

(Cortes et al., [1999])

M TD : $150 \mathrm{mg} / \mathrm{m}^{2}$ D aunoX ome, Tage 1- 3

$D$ aunoX ome hat eine signifikante antileukämische A ktivität

\section{Was bringt eine höhere Dosisintensität?}

Die gepoolte A nalyse verschiedener multizentrischer A M L C G -Studien, die seit 1978 in D eutschland durchgeführt wurden, zeigt bei A M L-Patienten unter 60 Jahren, dass die D osisintensität der Induktionschemotherapie die R emissionsdauer beeinflusst. Je höher die Dosisintensität, desto länger bleiben die Patienten in der Remission.

Speziell jüngere Hochrisikopatienten mit einem hohen Serum-L D H (>700 U/I), komplexen K aryotypanomalien und mehr als 40\% Blastennachweis am Tag 16 profitieren von einer dosisintensivierten Behandlung, wie z.B. der TAD-HAM Induktionstherapie (Standarddosis gefolgt von einer Hochdosis) (A bb.4). Die Patienten überlebten signifikant länger als Patienten, die zweimal das TAD-Protokoll als Induktionstherapie erhalten hatten.

Die Frage war, ob dies auch für ältere Patienten gilt. Eine multizentrische randomisierte Studie der D eutschen A M L CG bei 344 A M L -Patienten $\geq 60$ Jahre bejaht dies. $D$ ie E rgebnisse zeigen bei diesen Patienten signifikante Prognosevorteile unter der $\mathrm{G}$ abe von $3 \times 60 \mathrm{mg} / \mathrm{m}^{2}$ D aunorubicin im Vergleich 


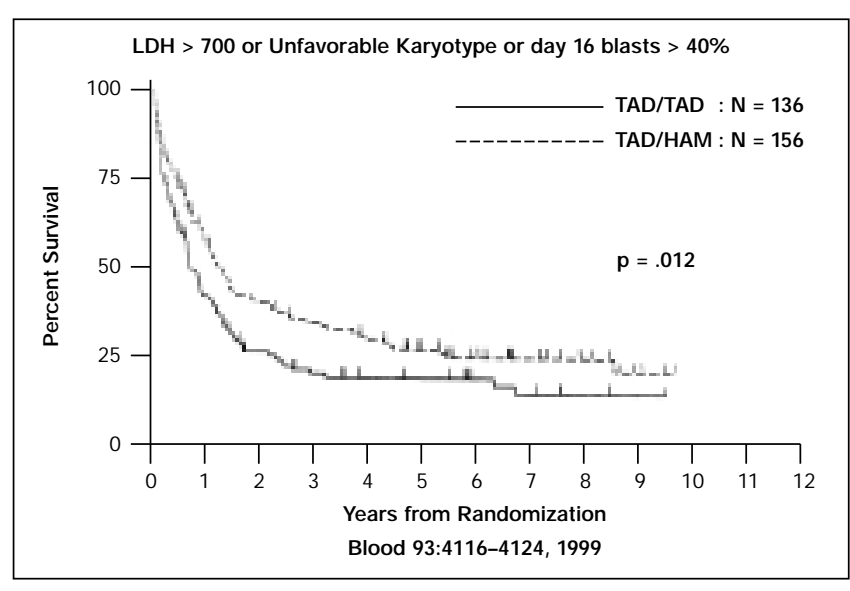

Abb. 4. $L$ ängere Ü berlebenszeiten bei prognostisch ungünstigen $A M L$ Patienten ( $\mathrm{LDH}>700 \mathrm{mg} / \mathrm{m}^{2} \mathrm{~K}$ aryotypanomalien, Blasten $>40 \%$ an Tag 16) unter intensivierter Chemotherapie (nach T. B üchner, M ünster).

zu der bislang üblichen D osierung von $3 \times 30 \mathrm{mg} / \mathrm{m}^{2}$ D aunorubicin, erläuterte B üchner. U nter der intensivierten Therapie sprachen mehr Patienten mit einer Remission an, und sie überlebten auch länger (A bb. 5).

Vor diesem $\mathrm{H}$ intergrund wird klar, dass die kumulative $\mathrm{K}$ ardiotoxizität der A nthrazykline an therapeutischer R elevanz gewinnen wird. Liposomale Darreichungsformen sind nicht nur eine wichtige Therapieoption für die Rezidivbehandlung, sondern möglicherweise auch für die First-lineB ehandlung.

\section{DaunoXome - eine interessante Option für ältere AML-Patienten}

Interview mit P rof. D r. T homas B üchner, U niversität M ünster

Was ist bei der $B$ ehandlung älterer Patienten mit rezidivierter AML zu beachten?

B üchner: Ä Itere Patienten haben überwiegend eine ungünstigere $\mathrm{K}$ rankheitsbiologie als jüngere Patienten. A ußerdem weisen sie oft Komorbidität auf, z. B. vorbestehende H erzerkrankungen, wodurch ihre Prognose zusätzlich verschlechtert wird.

$D$ ie ungünstige $K$ rankheitsbiologie ist nicht zwingend gleichbedeutend mit einem schlechteren Spontanverlauf der Erkrankung. Vielmehr führen ungünstige zytogenetische Veränderungen zu Chemotherapieresistenz. Zentrales Therapieziel ist es, diese Resistenzmechanismen zu überwinden. Die Strategie muss darin bestehen, wirksamere antileukämische Therapien einzusetzen und die Supportivtherapie zu verbessern.

Wiebewerten SieWirksamkeit und Verträglichkeit der D auno$X$ ome-M onotherapie bei Patienten mit rezidivierter $A M L$ ? B üchner: In unserer eigenen Phase I-Studie waren die Patienten bereits sehr einschlägig vorbehandelt und hatten kaum noch eine Chance, erfolgreich behandelt zu werden. Die E rgebnisse bestätigen der DaunoX ome-M onotherapie trotz dieser ungünstigen $\mathrm{R}$ andbedingungen eine geringe Toxizität. Wir haben keine wesentliche $\mathrm{K}$ ardiotoxizität gesehen, auch nicht auf der höchsten D osisstufe.
Dies deckt sich mit den E rgebnissen der U S-amerikanischen Phase-I-Studie, obwohl dieA merikaner deutlich höher dosierten. D osislimitierend war hier allerdings die M ukositis, so dass die maximal tolerable Dosis (MTD) auf $150 \mathrm{mg} / \mathrm{m}^{2}$ D auno$X$ ome an 3 konsekutiven Tagen festgelegt wurde. U nter dieser Dosierung traten keine schweren Nebenwirkungen auf. O ffenbar wird die $D$ aunoX ome-M onotherapie trotz beträchtlich hoher D osierung und trotz intensiver Vorbehandlung mit A nthrazyklinen gut vertragen.

A merikaner sprechen auch von einer signifikanten antileukämischen Wirkung. Wir können diese aufgrund unserer Studienergebnisse nicht bestätigen, allerdings war unsere Studie auch nicht daraufhin konzipiert worden. Wir haben mit einer relativ moderaten Dosierung eine gewisse palliative Wirkung bei stark vorbehandelten Patienten erzielt.

Welche Patienten können von $\mathrm{D}$ aunoX ome profitieren? $B$ üchner: Ich sehe die wirklichen Chancen von D aunoX ome nicht in der palliativen Therapiesituation. Zielgruppe für weitere Studien sind ältere A M L-Patienten im ersten R ezidiv. Sie sollten eine Kombinationstherapie mit $D$ aunoX ome plus A raC erhalten, die im Vergleich mit einer etablierten $\mathrm{R}$ ezidivtherapie zu testen ist. Z iel ist es, diese Patienten noch einmal in eine möglichst lang anhaltende R emission zu bekommen.

Welche D osierung bzw. welches Schedule empfehlen Sie? B üchner: D ie Dosierung sollte im obersten Bereich liegen, also $90-150 \mathrm{mg} / \mathrm{m}^{2}$ DaunoX ome an den Tagen 1-3. Das 3-Tage-Schedule bietet sich für die Kombinationsbehandlung mit A raC im $\mathrm{R}$ ahmen einer vergleichenden Studie mit konventionell appliziertem $D$ aunorubicin an. Die genaue $D$ osis muß man noch diskutieren, aber mit $150 \mathrm{mg} / \mathrm{m}^{2}$ D aunoX ome an 3 Tagen gibt es jetzt zumindest eine D osisvorstellung für eine Therapie in Kombination mit A raC. I ch persönlich halte $D$ aunoX ome für eines der interessantesten M edikamente, die wir derzeit für Phase-II -Studien bei A M L -Patienten im ersten R ezidiv für die Kombinationstherapie haben.

\section{CDaXOP: DaunoXome in Kombination mit Cyclophosphamid, Vincristin und Prednison beim hochmalignen bzw. intermediären Non-Hodgkin-Lymphom (NHL)}

Das CHOP-R egime (Cyclophosphamid, Doxorubicin, Vincristin, Prednison) ist die Standardtherapie für die Behandlung aggressiver Lymphome. Durch den Austausch von Doxorubicin durch liposomales $D$ aunorubicin soll die Verträglichkeit des $\mathrm{R}$ egimes verbessert werden.

Doxorubicin ist eine wesentliche Komponente des $\mathrm{CHOP}$ Regimes. Um die Verträglichkeit der Behandlung zu verbessern, speziell die Gefahr der Anthrazyklin-induzierten kumulativen Kardiotoxizität zu reduzieren, und gleichzeitig eine höhere Wirkstoffkonzentration im Tumorgewebe zu erreichen, haben Röhnisch/Hallek et al., Klinikum G roßhadern, M ünchen, im Rahmen einer Phase-I/II-Studie das Doxorubicin innerhalb des CHOP-Regimes durch liposomales $D$ aunorubicin ( $D$ aunoX ome) ersetzt.

Es ist bekannt, dass liposomales Daunorubicin eine veränderte Pharmakokinetik hat, erläuterte Prof. Dr. med. $M$ ichael Hallek, K linikum G roßhadern, M ünchen: $D$ ie Plasma-H albwertszeit ist verlängert, das Verteilungsvolumen 


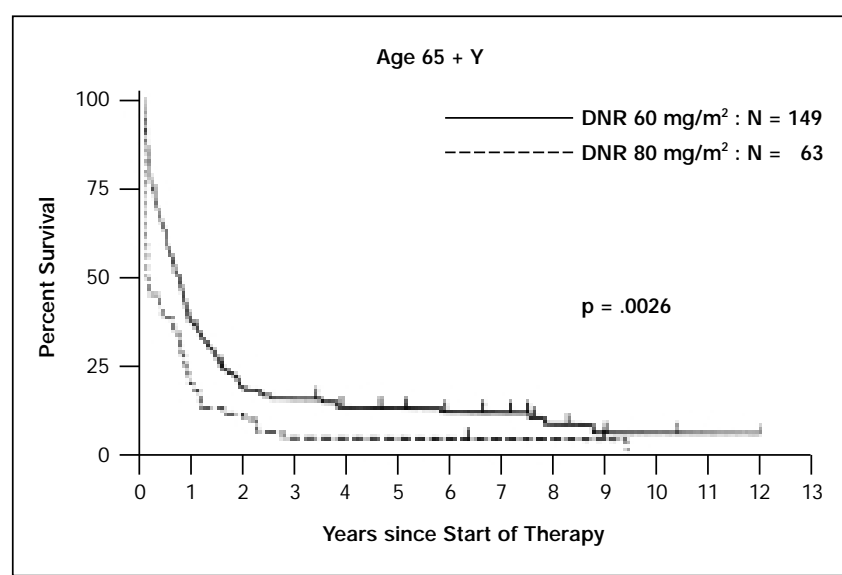

Abb. 5. L ängere Ü berlebenszeiten älterer A M L -Patienten ( $\geq 65$ Jahre) unter intensivierter Daunorubicin-M onotherapie (nach T. Büchner, M ünster).

im normalen $\mathrm{G}$ ewebe geringer und die $\mathrm{A}$ kkumulation des Z ytostatikums im Tumorgewebe erhöht. A Is $\mathrm{H}$ ypothese ergibt sich daraus, dass die Behandlung mit liposomalem D aunorubicin wirksamer ist und weniger Nebenwirkungen induziert. Letzteres impliziert die Möglichkeit einer Dosissteigerung und damit einer weiteren Erhöhung der Wirksamkeit.

Vorausgegangene klinische Studien haben gezeigt, dass unter $D$ aunoX ome weniger Nebenwirkungen auftreten als unter konventionell appliziertem A nthrazyklin. Selbst bei hohen kumulativen Dosen von $>1000 \mathrm{mg} / \mathrm{m}^{2}$ bleiben beispielsweise die kardialen F unktionen unverändert (A bb. 6). Dies ist nicht nur unter palliativen, sondern auch unter kurativen $G$ esichtspunkten ein wichtiger A spekt.

\section{CDaXOP - eine wirksame und gut verträgliche Alternative}

Endpunkte der M ünchner Studie waren die dosislimitierenden Toxizitäten, die maximal tolerable D osis sowie die E valuierung des $\mathrm{N}$ ebenwirkungsspektrums und der Wirksamkeit des modifizierten CHOP-R egimes, CDaXOP, bei Patienten mit rezidiviertem $\mathrm{NHL}$.

$D$ ie wichtigsten E inschlusskriterien der Studien:

- rezidiviertes NHL (kein lymphoblastisches NH L),

- Therapieintervall zur letzten CHOP-Chemotherapie mindestens $1 \mathrm{Jahr}$,

- A Iter der Patienten: 18-75 Jahre,

- A llgemeinzustand: E COG 2.

- Schriftliche E inverständniserklärung,

- Stadium II-IV (A nn A rbor).

Wichtigste A usschlusskriterien waren schwere kardiale Vorerkrankungen sowie eine kumulative $A$ nthrazyklin-G esamtdosis (ein oder zwei A nthrazykline) von $>60 \%$ der empfohlenen D osis. D ie Patienten mussten auf eine initiale CHOPTherapie angesprochen haben .

Tabelle 6 zeigt den Behandlungsplan. Bei fixen Standarddosierungen von Cyclophosphamid, Vincristin und Prednison wurde DaunoX ome in drei verschiedenen Dosen appliziert: $60-80-100 \mathrm{mg} / \mathrm{m}^{2}$ (Tag 1). In die Studie wurden 19 Patienten mit einem medianen A Iter von 62 Jahren aufgenommen. Es

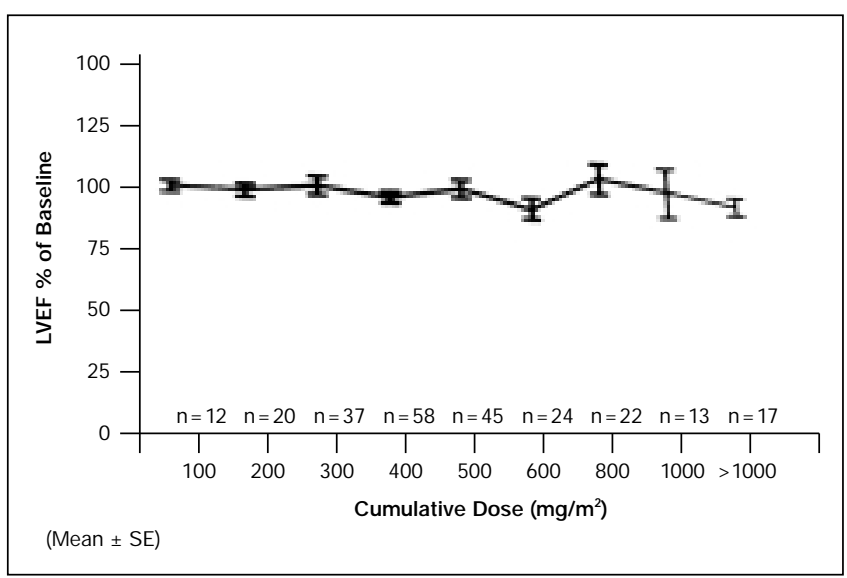

Abb. 6. Ergebnisse kontrollierter Studien zeigen, dass die LVEF unverändert bleibt unter DaunoX ome ${ }^{\circledR}$, selbst bei Dosen $>1000 \mathrm{mg} / \mathrm{m}^{2}$ (nach M. H allek, M ünchen).

handelte sich um typische R ezidivpatienten, meist mit einem follikulären Lymphom $(1 \times$ MALT, $9 \times \mathrm{cb} / \mathrm{cc}, 7 \times \mathrm{cb}, 1 \times \mathrm{cc}$, $1 \times A$ ILD ). Die M ehrzahl der Patienten (79\%) befand sich im Stadium III/IV. 7/19 Patienten waren mit CH O P vorbehandelt. R elativ wenige Patienten hatten erhöhte LD H -Werte im Plasma.

D ie N ebenwirkungen zeigt Tabelle 7. In den beiden niedrigen D osisstufen traten keine G rad-4- und nur sehr selten G rad-3N ebenwirkungen auf. In der höheren D osisstufe $\left(100 \mathrm{mg} / \mathrm{m}^{2}\right)$ war die G rad-4-L eukopenie dosislimitierend. Der Verlauf des Leukozytennadirs zeigt, dass es relativ schnell wieder zu einem A nstieg der L eukozyten kam, die L eukopenie also nur von kurzer $\mathrm{D}$ auer war ( $\mathrm{A}$ bb. 7). A nämien und Thrombozytopenien nahmen dosisabhängig zu, erreichten aber nicht den Schweregrad 4.

$\mathrm{N}$ ichthämatologische $\mathrm{N}$ ebenwirkungen waren laut $\mathrm{H}$ allek nur leicht ausgeprägt. Sehr selten kam es zur A lopezie. - D ie maximal tolerable D osis betrug $80 \mathrm{mg} / \mathrm{m}^{2} \mathrm{D}$ aunoX ome im $\mathrm{R}$ ahmen des CD aX OP-R egimes.

A Is erfreulich hoch bezeichnete $\mathrm{H}$ allek die A nsprechrate mit 9 kompletten und 5 partiellen Remissionen. Die objektive R emissionsrate betrug 74\% (Tab. 8). 6 von 7 Patienten, die mit $\mathrm{CHOP}$ vorbehandelt waren, erreichten erneut eine objektive R emission $(3 \times C R, 3 \times P R)$. Trotz der kleinen Fallzahlen sind dies laut Hallek sehr ermutigende Zahlen, die in weiteren Studien validiert werden sollten.

Fazit: Das CDaXOP-Regime kann problemlos appliziert werden, ohne dass schwere N ebenwirkungen auftreten. D osislimitierende Nebenwirkungen traten ab einer DaunoX omeDosis von $100 \mathrm{mg} / \mathrm{m}^{2}$ bei $4 / 7$ Patienten auf. Die maximal tolerable Dosis liegt daher bei $80 \mathrm{mg} / \mathrm{m}^{2}$ DaunoX ome im $R$ ahmen des CD aX O P-R egimes. In dieser D osierung war das R egime erstaunlich wirksam und wurde gut vertragen, obwohl es sich um rezidivierte Patienten handelte.

Die typischen A nthrazyklin-assoziierten Nebenwirkungen, wie z.B. K ardiotoxizität, wurden nicht oder kaum beobachtet. $\mathrm{Die} R$ ate der kompletten $\mathrm{R}$ emissionen lag extrem hoch, deutlich höher als normalerweise unter dem CH O P-R egime in der First-line-B ehandlung. Wichtig ist, dass das $C D$ aX O P-R egime auch bei CHOP-refraktären Patienten wirksam zu sein scheint. 
Tab. 6. Behandlungsplan des CDaX OP-Protokolls (nach M. Hallek, M ünchen

\begin{tabular}{llll}
\hline & $\begin{array}{l}\text { Dosis } \\
\mathrm{mg} / \mathrm{m}^{2}\end{array}$ & Route & Tag \\
\hline Cyclophosphamid & 750 & i.v. & 1 \\
DaunoX ome & $60-80-100$ & i.v. & 1 \\
Vincristin & $1,4^{\mathrm{a}}$ & i.v. & 1 \\
Prednison & 100 & per os & $1-5$ \\
\hline
\end{tabular}

Pro D osisstufe: 6 Patienten.

D osissteigerung: keine dosislimitierende Toxizität bei mehr als 3/6

Patienten.

a Max. $2 \mathrm{mg}$.

Tab. 7. Nebenwirkungen unter dem CD aX O P-R egime (nach M. Hallek, $M$ ünchen

\begin{tabular}{|c|c|c|c|c|c|c|c|c|c|c|c|c|}
\hline \multirow[b]{2}{*}{ WHO-Grad } & \multicolumn{4}{|c|}{$\begin{array}{l}\text { Dosis-L evel 1: } \\
60 \mathrm{mg} / \mathrm{m}^{2} \mathrm{DaX}\end{array}$} & \multicolumn{4}{|c|}{$\begin{array}{l}\text { Dosis-L evel 2: } \\
80 \mathrm{mg} / \mathrm{m}^{2} \mathrm{DaX}\end{array}$} & \multicolumn{4}{|c|}{$\begin{array}{l}\text { Dosis-L evel 3: } \\
100 \mathrm{mg} / \mathrm{m}^{2} \mathrm{DaX}\end{array}$} \\
\hline & I & II & III & IV & I & II & III & IV & I & II & III & IV \\
\hline Leukopenie & - & 5 & 1 & - & - & 1 & 5 & - & - & - & 2 & 4 \\
\hline A nämie & - & - & - & - & 2 & - & - & - & 2 & 3 & 1 & - \\
\hline Thrombozytopenie & - & - & - & - & - & - & - & - & 1 & 3 & - & - \\
\hline E rbrechen & 1 & 1 & 1 & - & 1 & 1 & 1 & - & - & 2 & 1 & - \\
\hline Mukositis & 3 & - & - & - & 1 & 1 & - & - & 4 & 2 & - & - \\
\hline A lopezie & 1 & 2 & - & - & 5 & 1 & - & - & 2 & 2 & 1 & - \\
\hline \multicolumn{13}{|l|}{ Toxizität } \\
\hline Kardiale & 1 & - & - & - & - & - & - & - & - & 1 & - & 1 \\
\hline Neurologische & 1 & - & 1 & - & - & 1 & - & - & 1 & 2 & - & 1 \\
\hline Gastrointestinale & - & 1 & - & - & 1 & 1 & - & - & 2 & 2 & - & - \\
\hline A rthralgie & - & 1 & 2 & - & - & 1 & - & - & - & 1 & - & - \\
\hline Infektion & - & - & 1 & - & 1 & - & - & - & - & 2 & - & - \\
\hline
\end{tabular}

\section{CDaXOP - ein Regime mit Zukunft beim hochmalignen NHL}

\section{Interview mit Prof. D r. med. M ichael H allek, M ünchen}

Warum haben Sie in dem Standardprotokoll $\mathrm{CHOP}$ das $D$ oxorubicin durch liposomales $D$ aunorubicin ersetzt?

$H$ allek: Z wei Ü berlegungen haben eine R olle gespielt: Es gab $\mathrm{H}$ inweise darauf, dass die Toxizität, speziell die K ardiotoxizität, unter liposomalem $D$ aunorubicin niedriger und dass die zytotoxische Wirksamkeit höher ist als die von D oxorubicin. $M$ it beiden Eigenschaften verbindet sich die Hoffnung, die zytostatische A ktivität des CHOP-Regimes zu erhöhen. Gerade bei Patienten mit hochmalignen Lymphomen und hohem R ezidivrisiko wäre dies wünschenswert.

Wie beurteilen Sie aus Ihrer klinischen E rfahrung die Wirksamkeit des modifizierten CH O P-Protokolls, des CD aX OPRegimes, im Vergleich zum klassischen CH OP-P rotokoll? Hallek: Ich halte das CDaXOP-R egime für mindestens genauso wirksam wie das CH O P-R egime. D arauf weisen jedenfalls unsere Studienergebnisse hin. Wir hatten in unserer Studie einen hohen Prozentsatz an Patienten mit follikulären Lymphomen. Normalerweise spricht gut die $\mathrm{H}$ älfte dieser

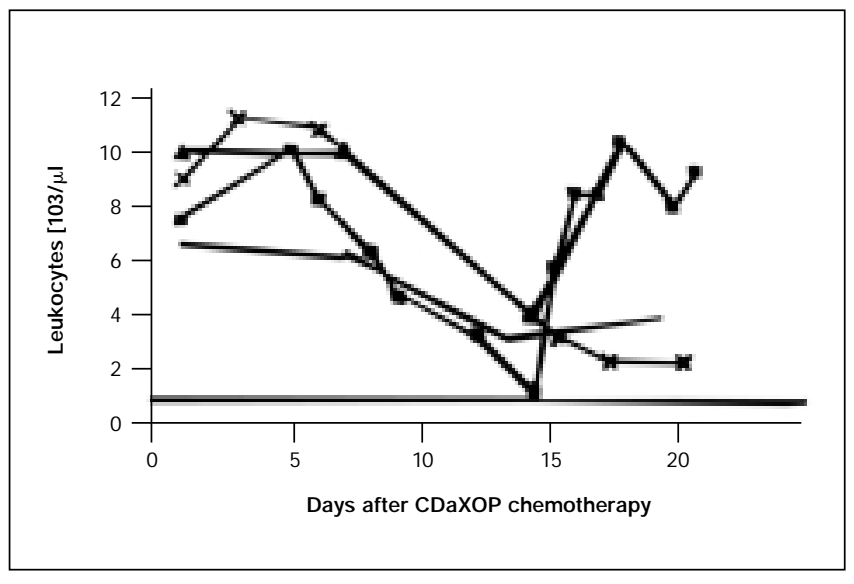

Abb. 7. Verlauf der Leukozytopenie mit Leukozytennadir nach CD aX O P-Polychemotherapie (nach: M. H allek, M ünchen).

Tab. 8. A nsprechraten nach CD aX O P-Polychemotherapie bei 19 Patienten mit rezidiviertem hochmalignem bzw. intermediärem NHL (nach M. H allek, M ünchen)

\begin{tabular}{lrr}
\hline & \multicolumn{2}{c}{ Patienten } \\
\cline { 2 - 3 } & $\mathrm{n}$ & $\%$ \\
\hline Komplette R emission (CD) & $9 / 19$ & 47 \\
Teilremission (PR) & $5 / 19$ & 26 \\
Objektive Remissionen (CR +PR) & $14 / 19$ & 74 \\
Tumorstabilisierung (NC) & $2 / 19$ & \\
Progress (PD) & $3 / 19$ & \\
\hline
\end{tabular}

Patienten in der Rezidivbehandlung noch einmal auf das CHOP-R egime mit einer objektiven R emission an. In unserer Studie betrug die objektive R emissionsrate jedoch fast $75 \%$. Dieses ermutigende Ergebnis sollte jetzt in einer großen Studie validiert werden.

$\mathrm{H}$ aben Sie eine $\mathrm{E}$ rklärung für diese gute Wirksamkeit? $\mathrm{H}$ allek: D iese ließe sich mit einer selektiven A nreicherung der L iposomen in den Lymphomen erklären. Im Tierexperiment wurde die $A$ kkumulation von $D$ aunorubicin im Tumorgewebe nach liposomaler A pplikation gezeigt. D ies ist eine der interessantesten Eigenschaften des liposomalen Daunorubicins. Der exakte M echanismus dieser Beobachtung ist noch nicht bekannt. A us diesem G rund und aus der Ü berlegung heraus, diesen $\mathrm{E}$ ffekt therapeutisch optimal zu nutzen, halte ich es für wichtig, entsprechende U ntersuchungen auch am Patienten durchzuführen, z. B. mit den neuen bildgebenden Verfahren.

G ibt es prognostische Parameter, welche Patienten besonders gut auf CD aX OP ansprechen?

H allek: U nsere Subgruppenanalyse hat ergeben, dass Patienten mit einem hohen LDH-Wert nicht gut ansprechen. A lle anderen Patienten haben angesprochen.

Wie bewerten Sie das $\mathrm{N}$ ebenwirkungsspektrum von $\mathrm{CD}$ aX OP? $\mathrm{H}$ allek: U ns fiel auf, dass die Patienten unter CD aX OP kaum eine $A$ lopezie entwickelten. Das ist psychologisch ein ganz wichtiger Punkt und trägt deutlich dazu bei, dass die Patienten 
die Therapie auch subjektiv gut vertragen. $D$ ie gute kardiale Verträglichkeit bestätigte sich. W ir hatten keine $\mathrm{H}$ inweise darauf, dass sich die linksventrikuläre Auswurffraktion unter CDaX OP veränderte. Ebenfalls wichtig: Es kam kaum zu Ü belkeit und E rbrechen.

Welche E rfahrung haben Sie mit der ambulanten $\mathrm{G}$ abe von CDaXOP?

$\mathrm{H}$ allek: D as CD aX O P-R egime ist problemlos ambulant durchführbar. E ine stationäre B ehandlung ist normalerweise nicht notwendig.

Welche Dosierung empfehlen Sie für D aunoX ome innerhalb des CD aX O P-R egimes?

$\mathrm{H}$ allek: D ie maximal tolerable D osis in unserer Studie betrug $80 \mathrm{mg} / \mathrm{m}^{2} \mathrm{D}$ aunoX ome in Kombination mit den etablierten D osierungen der anderen Zytostatika dieses R egimes. D iese D osierung empfehle ich auch für weitere klinische Studien.

Welche Patienten sollten CD aX OP erhalten?

$\mathrm{H}$ allek: Der therapeutische Stellenwert von CDaXOP muss in klinischen Studien weiter validiert werden. A ußerhalb klinischer Studien sehe ich zur Zeit in CDaX OP eine gute Therapiealternative für rezidivierende und für ältere Patienten. Langfristig besteht die Ü berlegung, CDaXOP in der Primärbehandlung einzusetzen. Dies muss aber erst noch in entsprechenden klinischen Studien überprüft werden.

Welche weiteren Studien sind mit CD aX O P geplant?

$\mathrm{H}$ allek: D ie D eutsche Non-H odgkin-Lymphom-G ruppe plant eine größere Phase-II-Studie bei Patienten mit niedrig-malignem NHL. In der Deutschen CL L -Studiengruppe überlegen wir, die 3er-Kombination Fludarabin/M itoxantron/C yclophosphamid zu modifizieren und das M itoxantron durch Dauno$X$ ome zu ersetzen. $D$ ie 3er-Kombination ist derzeit die wirksamste Therapie für CLL-Patienten, allerdings ist das M itoxantron - ein A nthrazyklin-ähnliches Zytostatikum - sehr myelotoxisch. DaunoX ome könnte eine besser verträgliche A Iternative sein.

E inen interessanten A nsatz sehe ich in der Intensivierung des CD aX O P-R egimes in der Kombination mit Wachstumsfaktoren. Dies könnte die Basis für ein Hochdosiskonzept als Primärtherapie sein. Hierzu ist aber meines Wissens derzeit keine Studie geplant.

\section{AmBisome: Liposomales Amphotericin B für Patienten mit febriler Neutropenie nach allogener Stammzelltransplantation}

Systemische Pilzinfektionen gehören zu den schwer wiegendsten Komplikationen in der klinischen Medizin. Sie können lebensbedrohlich werden, wenn keine adäquate B ehandlung erfolgt. Therapie der Wahl ist das A ntimykotikum A mphotericin B. Klinisch problematisch und daher immer wieder Therapie-limitierend sind allerdings die oft signifikanten Nebenwirkungen, speziell die Nephrotoxizität. K linische Studien zeigen, dass liposomales A mphotericin B (A mB isome) deutlich besser verträglich ist als das konventionell applizierte A mphotericin B, ohne dass die hohe Wirksamkeit der Substanz eingeschränkt ist, erläuterte PD Dr. Pablo Cagnoni, D enver/USA.
Systemische Pilzinfektionen nehmen unter anderem aufgrund immer aggressiverer Chemotherapien in der O nkologie sowie vermehrt durchgeführter Transplantationen zu. Kommt es nach einer $\mathrm{K}$ nochenmark- oder Stammzelltransplantation zu Infektionen, entwickeln sie sich im allgemeinen innerhalb der ersten 4 Wochen in der neutropenischen Phase der Patienten. In diversen klinischen Studien zeigte sich, dass A mBisome nach $\mathrm{K}$ nochenmarktransplantation gut vertragen wird - auch in höherer D osis von täglich $5 \mathrm{mg} / \mathrm{kg} \mathrm{K}$ örpergewicht. N ebenwirkungen waren selten. W ichtig: E s traten keine signifikanten $\mathrm{N}$ ierenschädigungen auf, und es zeigte sich keine signifikante Verschlechterung der $\mathrm{H}$ arnstoff- und $\mathrm{K}$ reatininwerte im Serum.

Die gute Verträglichkeit von liposomalem A mphotericin B ( $A$ mB isome) bestätigte sich auch bei neutropenischen Patienten nach allogener Stammzelltransplantation. Cagnoni stellte die E rgebnisse von zwei multizentrischen und randomisierten Doppelblinduntersuchungen vor.

\section{Multizentrische randomisierte Doppelblind- Studie mit AmBisome versus konventionelles Amphotericin B}

Die erste $A$ nalyse basiert auf den D aten von 103 neutropenischen Patienten mit allogener Stammzelltransplantation [Cagnoni et al., 1997], die im Rahmen einer doppelblind durchgeführten, multizentrischen, randomisierten Studie mit insgesamt 687 Patienten alternativ mit liposomalem bzw. konventionell appliziertem A mphotericin B behandelt worden waren [Walsh et al., 1999]. Kommt es nach allogener Stammzelltransplantation trotz A ntibiotikagabe zu einer febrilen N eutropenie, so besteht ein hohes R isiko für eine systemische Pilzinfektion.

50 Patienten erhielten eine Behandlung mit konventionell appliziertem A mphotericin B, und 53 Patienten wurden mit der liposomalen Darreichungsform von A mBisome behandelt. Die beiden Patientengruppen waren hinsichtlich ihrer Charakteristika vergleichbar, betonte Cagnoni.

D as mediane A Iter betrug jeweils 34 Jahre. G ut zwei D rittel der Patienten hatten als A usgangsdiagnose eine akute oder eine chronische Leukämie. Weitere Diagnosen waren Lymphome, M yelodysplasien, refraktäre $A$ nämien oder $M$ yelome. Der K reatinin-A usgangswert betrug $0,75 \mathrm{mg} / \mathrm{dl}$ (A mB isome) bzw. 0,81 mg/dl. E in Teil der Patienten hatte eine FluconazolProphylaxe erhalten: $64 \%$ der Patienten im A mB isome-A rm und $50 \%$ im Vergleichsarm.

\section{Verträglichkeit und Wirksamkeit signifikant verbessert}

Die Ergebnisse der A nalyse bestätigen dem liposomalen A mphotericin B nicht nur eine signifikant geringere Toxizität, sondern auch eine bessere Wirksamkeit. D ie R ate an N ephrotoxizitäten, definiert als Verdoppelung des Kreatinin-A usgangswertes, war unter konventionell appliziertem A mphotericin $B$ mehr als doppelt so hoch wie unter der liposomalen Gabe: 66 vs. 32\%. D ieser U nterschied war statistisch signifikant ( $p<0,001)$ (Tab. 9).

A ußerdem wurden im Standardarm signifikant häufiger infusionsbedingte N ebenwirkungen beobachtet. $\mathrm{D}$ ie $\mathrm{H}$ älfte derjenigen Patienten, die konventionell appliziertes A mphoteri- 
Tab. 9. E rgebnisse im Vergleich: liposomales A mphotericin B (A mBisome) versus konventionell appliziertes A mphotericin B bei Patienten nach allogener Stammzelltransplantation [Cagnoni et al., 1997]

\begin{tabular}{|c|c|c|c|c|c|}
\hline & \multicolumn{2}{|c|}{ A mB isome } & \multicolumn{2}{|c|}{$\begin{array}{l}\text { Konventionell appliziertes } \\
\text { A mphotericin B }\end{array}$} & \multirow[t]{2}{*}{$p$-Wert } \\
\hline & $\mathrm{n}$ & $\%$ & $\mathrm{n}$ & $\%$ & \\
\hline \multicolumn{6}{|l|}{ Verdoppelung des } \\
\hline A usgangskreatininwertes & & 32 & & 66 & $>0,001$ \\
\hline $\mathrm{H}$ ämodialyse & 1 & & 5 & & 0,08 \\
\hline \multicolumn{6}{|l|}{ H ypokaliämie } \\
\hline$<3,0 \mathrm{mval} / \mathrm{l}$ & & 19 & & 14 & 0,51 \\
\hline D osisreduktionen & & 17 & & 60 & $<0,001$ \\
\hline
\end{tabular}

cin B erhielten, hatte am 1. Therapietag einen Fieberschub $\geq 1,0^{\circ} \mathrm{C}$ im Vergleich zu $2 \%$ der Patienten im A mBisomeA rm. Nur $17 \%$ der Patienten, die liposomales A mphotericin B erhalten hatten, benötigten D osisreduktionen, im Vergleich zu $60 \%$ der Patienten im konventionellen A rm ( $p<0,001)$. K eine signifikanten U nterschiede zwischen beiden Therapiearmen zeigten sich bei der $\mathrm{H}$ epatotoxizität.

Im A mB isome-A rm kam es signifikant seltener zu einem erneuten Durchbruch von Pilzinfektionen: 2 versus $14 \%$ $(p=0,05)$ (Tab. 10). Tabelle 11 zeigt die E rgebnisse der 8 Patienten, bei denen eine Pilzinfektion trotz A mphotericin-B Therapie auftrat: B ei 5 der 7 Patienten, die konventionelles A mphotericin B erhalten hatten, hatte die Dosis reduziert werden müssen. Dies bestätigt die hohe Toxizität von A mphotericin $\mathrm{B}$ als $\mathrm{H}$ auptproblem der B ehandlung, betonte Cagnoni.

Fazit: A mB isome ist bei neutropenischen Patienten mit allogener Stammzelltransplantation signifikant besser verträglich und wirksamer als konventionell appliziertes A mphotericin $B$.

\section{Multizentrische randomisierte Doppelblind- Studie mit AmBisome versus ABLC-Behandlung}

Die gute Verträglichkeit des liposomalen A mphotericin B (A mBisome) bestätigte sich in einer weiteren multizentrischen, randomisierten Doppelblindstudie [Wingard et al., 2000]. Patienten mit febriler N eutropenie erhielten alternativ liposomales A mphotericin B bzw. A B L C. DieA BL C-Behandlung gilt als relativ toxisch. Im M ittelpunkt der Studie stand daher die Therapiesicherheit.

In diese 3-armige Studie wurden mehrheitlich N iedrig-R isikoPatienten mit akuter Leukämie, einem soliden Tumor oder einem Lymphom als A usgangsdiagnose aufgenommen. U ngefähr die $\mathrm{H}$ älfte der Patienten hatte eine $\mathrm{K}$ nochenmarktransplantation erhalten. A Iternativ erhielten die Patienten $(n=80)$ A BLC (A mphotericin B Lipid Komplex), 5 mg/kg/Tag oder liposomales A mphotericin B in zwei unterschiedlichen D osierungen, nämlich $5 \mathrm{mg} / \mathrm{kg} / \mathrm{Tag}(\mathrm{n}=83)$ oder $3 \mathrm{mg} / \mathrm{kg} / \mathrm{Tag}$ $(n=87)$.

\section{Signifikant niedrigere Nephrotoxizitätsrate}

D ie A nsprechrate lag in den beiden Therapiearmen mit liposomalem A mphotericin B am höchsten, ohne klinisch rele-
Tab. 10. E rneutes A uftreten systemischer Pilzinfektionen unter antimykotischer B ehandlung mit A mphotericin B [Cagnoni et al., 1997]

\begin{tabular}{lll}
\hline Patienten mit allogener KMT & \multicolumn{2}{l}{ Infektionen } \\
\cline { 2 - 3 } & $\mathrm{n}$ & $\%$ \\
\hline Liposomales A mphotericin B (A mB isome) & 1 & $1,9 \% \quad(p<0,05)$ \\
Konventionell appliziertes A mphotericin & 7 & 14,0 \\
\hline
\end{tabular}

A uswertung durch ein unabhängiges R eview-Komitee.

vante Unterschiede zwischen beiden Dosierungen. Die A nsprechrate für beide A rme betrug $41 \%$, versus $33 \%$ im A B L C-Vergleichsarm.

E ine analoge Situation zeigte sich bei den Nebenwirkungen bzw. bei der $\mathrm{R}$ ate an Therapieabbrüchen: B eide $A$ mphotericin$B$-A rme waren der A B L C-B ehandlung signifikant überlegen, ohne dass klinisch relevante $U$ nterschiede zwischen beiden D osierungen bestanden. In 32\% der Fälle musste die A B L C Behandlung abgebrochen werden, im Vergleich zu 13\% der Patienten, die liposomales A mphotericin B erhalten hatten. U rsachen dafür waren eine - zum Teil statistisch signifikant höhere Rate an Schüttelfrost, Fieber, Hypoxie und ein erhöhter Serumkreatinin-Wert im A BL C-A rm (Tab. 12).

Besonders deutlich waren die U nterschiede bei der Nephrotoxizität: 42\% der Patienten im A B L C-A rm zeigten eine Verdopplung des A usgangs-Serumkreatinin-Wertes im Vergleich zu $15 \%$ der Patienten, die liposomales A mphotericin B erhalten hatten. Signifikante U nterschiede ergaben sich auch beim Vergleich der jeweiligen Spitzenkreatinin-Werte unter A B L C - bzw. liposomaler A mphotericin-B-Therapie.

\section{Pharmakoökonomische Analyse: AmBisome ist kosteneffektiv}

Die Medikamentenkosten für liposomales A mphotericin B liegen höher als diejenigen für konventionelles $A$ mphotericin B [Cagnoni et al., 2000]. D as heißt jedoch nicht, dass die G esamtbehandlung deutlich teurer und die Therapie mit liposomalem A mphotericin B nicht kosteneffektiv wäre, betonte Cagnoni. U m dies zu prüfen, haben Cagnoni et al. eine retrospektive pharmakoökonomische U ntersuchung durchgeführt. 
Tab. 11. N achgewiesene systemische Pilzinfektionen unter antimykotischer B ehandlung mit A mphotericin $B$ [Cagnoni et al., 1997]

\begin{tabular}{|c|c|c|c|c|c|}
\hline & E rreger & Lokalisation & $\begin{array}{l}\text { Flukonazol- } \\
\text { Prophylaxe }\end{array}$ & $\begin{array}{l}\text { Dosis- } \\
\text { reduktion }\end{array}$ & $\begin{array}{l}\text { Todes- } \\
\text { ursache }\end{array}$ \\
\hline \multirow{7}{*}{$\begin{array}{l}\text { Konventionell } \\
\text { appliziertes } \\
\text { A mphotericin B } \\
(n=7)\end{array}$} & A . fumigatus & Sinus & ja & ja & nein \\
\hline & C. albicans & Blut & nein & nein & nein \\
\hline & C. krusei & Blut & nein & nein & ja (C. krusei) \\
\hline & A.spp. & Bronchien & nein & ja & ja (A . spp.) \\
\hline & $\begin{array}{l}\text { H efe (nicht } \\
\text { spezifiziert) }\end{array}$ & Blut & nein & ja & ja (H efe) \\
\hline & C. glabrata & Blut & ja & ja & nein \\
\hline & $\begin{array}{l}\text { Fungi (nicht } \\
\text { spezifiziert) }\end{array}$ & Bronchien & nein & ja & ja (Fungi) \\
\hline A mBisome $(n=1)$ & Mucor & Bronchien & nein & nein & ja (M ucor) \\
\hline
\end{tabular}

\begin{tabular}{|c|c|c|c|c|c|c|}
\hline \multirow[t]{3}{*}{ E reignisse } & \multicolumn{4}{|c|}{ A mB isome } & \multirow{2}{*}{\multicolumn{2}{|c|}{$\begin{array}{l}\text { A BLC } \\
5 \mathrm{mg} / \mathrm{kg}(\mathrm{n}=78)\end{array}$}} \\
\hline & \multicolumn{2}{|c|}{$3 \mathrm{mg} / \mathrm{kg}(\mathrm{n}=85)$} & \multicolumn{2}{|c|}{$5 \mathrm{mg} / \mathrm{kg}(\mathrm{n}=81)$} & & \\
\hline & $\bar{n}$ & $\%$ & $\bar{n}$ & $\%$ & $\mathrm{n}$ & $\%$ \\
\hline $\begin{array}{l}\text { Therapieabbrüche } \\
\text { insgesamt }\end{array}$ & 11 & 12,9 & 10 & 12,3 & 25 & 32,1 \\
\hline Schüttelfrost & 1 & 1,2 & 1 & 1,2 & 5 & 6,4 \\
\hline Fieber & 0 & 0,0 & 1 & 1,2 & 8 & 6,4 \\
\hline E rhöhter K reatininwert & 2 & 2,4 & 1 & 1,2 & 8 & 10,3 \\
\hline H ypoxie & 1 & 1,2 & 0 & 0,0 & 6 & 7,7 \\
\hline
\end{tabular}

Tab. 12. Therapieabbrüche wegen $N$ ebenwirkungen. Vergleich von liposomalem A mphotericin B (A mB isome) in zwei D osierungen versus A B L C -Therapie [Cagnoni et al., 1997] zität unter konventioneller A mphotericin-B -Therapie, zusammenzuhängen. $F$ ür diese Patienten haben die $\mathrm{K}$ rankenhäuser in den USA deutlich mehr Kosten aufzuwenden. Im Vordergrund stehen zusätzliche supportive Therapiemaßnahmen. Die Kostendifferenz zugunsten der liposomalen Darreichungsform betrug 3,781 U S-\$ - bzw. 5,506 U S-\$ für die Subgruppe der Patienten mit allogener Stammzelltransplantation (A bb. 8a/b).

Fazit: Die beiden wichtigsten Kostenpunkte bei der B ehandlung mit A mphotericin B sind die M edikamentenkosten und die für nephrotoxische Komplikationen aufzuwendenden Kosten. Beide Faktoren müssen in die Kostenanalyse einfließen, betonte Cagnoni. Die Medikamentenkosten liegen unter liposomaler A mphotericin-B-Therapie höher, werden aber durch geringere Kosten für nephrotoxische Komplikationen relativiert. $\mathrm{D}$ ie A $\mathrm{mB}$ isome-Therapie ist kosteneffektiv, betonte Cagnoni. D ies gilt insbesondere unter Berücksichtigung der signifikant besseren Verträglichkeit für den Patienten. «In den U SA gilt: Nebenwirkungen kosten G eld», so das Fazit von Cagnoni.

\section{Patienten mit schweren Pilzinfektionen profitieren von liposomalem Amphotericin B}

Interview mit PD D r. med. Pablo Cagnoni, U niversität Colorado, D enver/USA

Welcher Zeitpunkt ist aufgrund Ihrer klinischen E rfahrung mit K nochenmark-transplantierten Patienten der geeignete für 

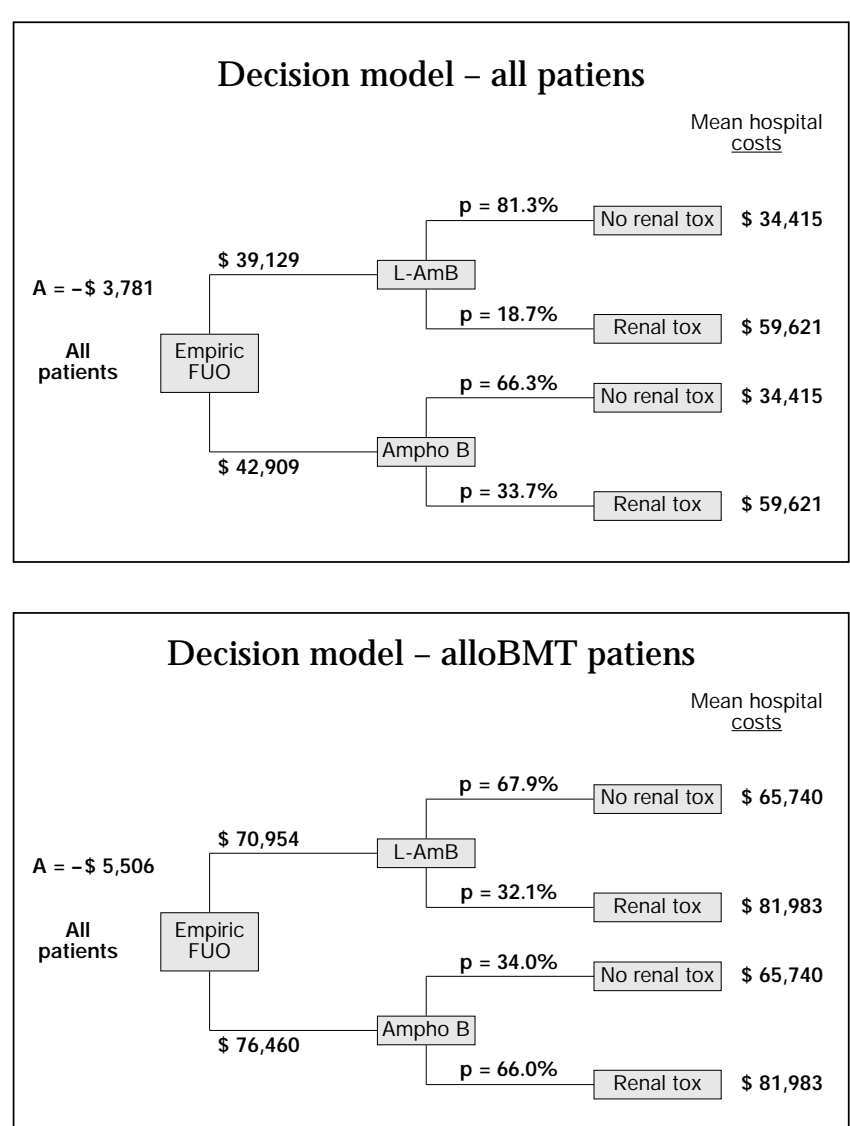

Abb. 8a/b. Entscheidungsmodell. Das Ausmaß der Nephrotoxizität beeinflusst die Kostensituation [C agnoni et al., 1997].

eine empirische antimykotische Therapie bei systemischen Pilzinfektionen? Welche D osierung empfehlen Sie?

Cagnoni: Eine empirische antimykotische Therapie sollte begonnen werden, wenn der Patient nach 96 Stunden nicht auf die Behandlung mit einem Breitspektrum-A ntibiotikum angesprochen hat. Hier deckt sich meine klinische E rfahrung mit den derzeit verfügbaren klinischen Studienergebnissen. A Is D osierung empfehle ich $0,6-0,8 \mathrm{mg} / \mathrm{kg}$ pro Tag A mphotericin B Deoxycholat bzw. $5 \mathrm{mg} / \mathrm{kg}$ pro Tag im Fall einer A BLC-Therapie. Die D osis von liposomalem A mphotericin B sollte bei H ochrisikopatienten $3 \mathrm{mg} / \mathrm{kg} / \mathrm{Tag}$ betragen.

Die B ehandlung mit konventionell appliziertem A mphotericin $B$ wird immer wieder durch schwere Nebenwirkungen limitiert, z. B. durch die Nephrotoxizität. Welche Patienten profitieren am deutlichsten von dem signifikant besseren Nebenwirkungsprofil des liposomalen A mphotericin B ?

Cagnoni: D en größten Benefit von liposomal appliziertem A mphotericin B haben Patienten nach allogener K nochenmarktransplantation, Patienten nach O rgantransplantation sowie Patienten, die eine langandauernde Behandlung benötigen, z.B. Patienten mit schweren invasiven Pilzinfektionen.

Wie bewerten Sie die Kosten-Effektivität von liposomalem A mphotericin B in der First-line-Behandlung bei Patienten mit febriler N eutropenie?
Cagnoni: Die Kosteneffektivität hängt damit zusammen, dass trotz der höheren M edikamentenkosten, die für liposomales A mphotericin $B$ anfallen, die Behandlung deutlich weniger toxisch ist als mit konventionellem A mphotericin B. In einigen Patientengruppen korreliert die zum Teil extensive Toxizität unter konventionellem A mphotericin B mit einem deutlichen Kostenanstieg aufgrund umfangreicher Supportivmaßnahmen.

Auf dem Markt sind verschiedene lipide A mphotericin-BFormulierungen. Worin sehen Sie die Hauptvorteile von A mB isome im Vergleich zu diesen Formulierungen?

Cagnoni: Eine randomisierte Studie, die gerade publiziert wird, hat gezeigt, dass liposomales A mphotericin B weniger nierenschädigend ist und weniger I nfusions-bedingte R eaktionen induziert als die ABLC-Behandlung. Das sind wesentliche Vorteile für das liposomale A mphotericin B. Das gilt besonders für die ambulante B ehandlung - auch deshalb, weil weniger Prämedikation benötigt wird. M eines Wissens ist das liposomale A mphotericin B bislang die einzige lipide Formulierung, für die gezeigt wurde, dass eine D osiseskalation bis zu $15 \mathrm{mg} / \mathrm{kg} / \mathrm{Tag}$ möglich ist, ohne dass Sicherheitsbedenken bestehen. D ie liposomale D arreichungsform hat die Pharmakokinetik von A mphotericin B verändert: Liposomales A mphotericin B erreicht höhere Spitzenkonzentrationen und hat eine längere $\mathrm{H}$ albwertszeit als A BLC. Welche klinische R elevanz dies hat, wissen wir noch nicht.

Wo sehen Sie die wichtigsten Herausforderungen für die Zukunft der antimykotischen Therapie, und welche R olle wird hier A mB isome spielen?

Cagnoni: Systemische Pilzinfektionen sind die $\mathrm{H}$ aupttodesursache bei K nochenmark-transplantierten Patienten, die an einer Infektion sterben. Es ist offensichtlich, dass wir wirksamere Therapien benötigen und die prophylaktischen Maßnahmen verbessern müssen. Die zentrale Herausforderung sehe ich in der E ntwicklung wirksamer Präventionsstrategien und/oder der Früherkennung. In den nächsten Jahren werden verschiedene neue antimykotische Substanzen zugelassen. E s wird wichtig sein, die Substanzen gegeneinander abzugrenzen und das jeweilige Indikationsgebiet exakt zu definieren. Liposomales A mphotericin B ist unabhängig davon eine wichtige Therapieoption, entweder als $\mathrm{M}$ onotherapie oder in $\mathrm{Kombi}$ nation mit einigen dieser neuen Substanzen, $z$. B. den E chinocandinen.

\section{Referenzliste}

Cagnoni $\mathrm{P}$, et al.: Pharmacoeconomic analysis of liposomal A mphotericin B versus conventional A mphotericin $B$ in the empirical treatment of persistently febrile neutropenic patients. J Clin O ncol 2000;18:2476-2483.

Cortes JE, et al.: Liposomal Daunorubicin ( $D$ aunoX ome) has minimal cardiotoxicity in patients with prior anthracycline therapy for A cute M yeloid L eukemia. Blood 1999;94(suppl 1):3276.

Michieli M, et al.: Liposome-encapsulated daunorubicin for PG P-related multidrug resistance. Br J H aematol 1999;106:92-99.

$\mathrm{R}$ öhnisch $\mathrm{T}$, et al.: Treatment of relapsed Non-H odgkin's-Lymphoma (NHL) with liposomal Daunorubicin (DaunoX ome) in combination with Cyclophosphamide, Vincristine and Prednisone (CD aX OP). B lood 2000;96:246B.

Walsh TJ, et al.: Liposomal A mphotericin B for empirial therapy in patients with persistent fever and neutropenia. N ew Engl J M ed 1999;340:764-771.

Wingard JR, et al.: A randomized, double-blind comparative trial evaluating the safety of liposomal A mphotericin B Lipid Complex in the empirical treatment of febrile neutropenia. Clin Infect D is 2000;31:1155-1163. 